\title{
Probióticos naturais para a prevenção e tratamento de doenças crônicas: Uma revisão
}

\author{
Natural probiotics for the prevention and treatment of chronic diseases: A review \\ Probióticos naturales para la prevención y tratamiento de enfermedades crónicas: Una revisión
}

Brena Lopes Miranda

ORCID: https://orcid.org/0000-0002-5083-1465 Universidade Federal do Piauí, Brasil

E-mail: b-rena13@hotmail.com

Kelly Maria Rêgo da Silva

ORCID: https://orcid.org/0000-0003-3070-4060 Laboratório Central de Saúde Pública do Piaú, Brasil

E-mail: kelly-rego@bol.com.br

Thalia Tauany de Sousa Barbosa

ORCID:https://orcid.org/0000-0002-9285-7460 Universidade Federal do Piauí, Brasil

E-mail: thaliatauany20@gmail.com

Diêgo De Oliveira Lima

ORCID: https://orcid.org/0000-0001-8211-9416

Universidade Federal do Piauí, Brasil

E-mail: di.oliveiralima@hotmail.com

Maria Lúcia de Moura Rocha Barbosa

ORCID: https://orcid.org/0000-0002-7633-9815 Universidade Federal do Piauí, Brasil

E-mail: mlesperanca@gmail.com

Juciara Carvalho de Oliveira

ORCID: https://orcid.org/0000-0002-2010-2587

Laboratório Central de Saúde Pública do Piauí, Brasil

E-mail: juciaracarvalho06@gmail.com

Higo José Neri da Silva

ORCID: https://orcid.org/0000-0003-2089-4147 Universidade Federal do Piauí, Brasil

E-mail: higoneri@gmail.com

Antony Charles Oliveira do Nascimento

ORCID: https://orcid.org/0000-0003-1862-9328

Técnologo em Radiologia, Brasil

E-mail: charlesnascimento.the@ hotmail.com

Suely Moura Melo

ORCID: https://orcid.org/0000-0001-9996-0850 Universidade Federal do Piauí, Brasil

E-mail: suelymouramelo@yahoo.com.br

Keylla da Conceição Machado

ORCID: https://orcid.org/0000-0002-4335-2829

Universidade Federal do Piauí, Brasil

E-mail:keyllamachado06@hotmail.com

Kivia Ludmilla Carvalho Ferreira

ORCID: https://orcid.org/0000-0003-4010-9927

DSEI Kaiapó do Pará, Brasil

E-mail: kivialudmilla@hotmail.com

Ag-Anne Pereira Melo de Menezes

ORCID: https://orcid.org/0000-0003-2830-990X

Universidade Federal do Piauí, Brasil

E-mail: ag-anne@hotmail.com

Gabriel Franco de Sousa

ORCID: https://orcid.org/0000-0003-1208-1357

Universidade Estadual do Piauí/FACIME, Brasil

E-mail: gabrielfrannco@ hotmail.com

Kananda Pereira Almeida

ORCID: https://orcid.org/0000-0001-5735-5353

Universidade Federal do Piauí, Brasil

E-mail: kananda.almeida@hotmail.com

Amanda Miranda da Silva

ORCID: https://orcid.org/0000-0001-6476-4809 Universidade Federal do Piauí, Brasil miraanda.sa@gmail.com 


\title{
Resumo
}

Para a promoção e a proteção da saúde, a alimentação e nutrição são consideradas requisitos básicos, dado que elas possibilitam a afirmação do potencial de crescimento e desenvolvimento humano com qualidade de vida. Nos últimos anos a substituição de uma alimentação caseira e natural por alimentos industrializados, afetando a população em geral influenciando negativamente o processo de saúde/doença. Essa revisão pretende explanar como os probióticos pode afetar nas doenças crônicas. Utilizou-se bases científicas como Scielo, Pubmed e Periódicos, onde foram selecionados os artigos que mais se encaixam no tema. Esse trabalho mostrou a importância dos probióticos para as doenças crônicas e também a necessidade de novas pesquisas para mais confirmações científicas.

Palavras-chave: Doenças crônicas; Probióticos; Alimentação.

\begin{abstract}
For the promotion and protection of health, food and nutrition are considered basic requirements, since they make it possible to affirm the potential for human growth and development with quality of life. In recent years, the substitution of homemade and natural food for industrialized foods, affecting the general population, negatively influencing the health / disease process. This review aims to explain how probiotics can affect chronic diseases. Scientific bases such as Scielo, Pubmed and Periodicos were used, in which the articles that best fit the theme were selected. This work showed the importance of probiotics for chronic diseases and also the need for further research for further scientific confirmation.
\end{abstract}

Keywords: Chronic diseases; Probiotics; Food.

\section{Resumen}

Para la promoción y protección de la salud, la alimentación y la nutrición se consideran requisitos básicos, ya que permiten afirmar el potencial de crecimiento y desarrollo humano con calidad de vida. En los últimos años, la sustitución de alimentos caseros y naturales por alimentos industrializados, afectando a la población en general, incidiendo negativamente en el proceso salud / enfermedad. Esta revisión tiene como objetivo explicar cómo los probióticos pueden afectar las enfermedades crónicas. Se utilizaron bases científicas como Scielo, Pubmed y Periodicos, en las que se seleccionaron los artículos que más se adaptaban a la temática. Este trabajo mostró la importancia de los probióticos para las enfermedades crónicas y también la necesidad de más investigación para una mayor confirmación científica.

Palabras clave: Enfermedades crónicas; Probióticos; Comida.

\section{Introdução}

Uma alimentação saudável deve conter alimentos funcionais, ricos em nutrientes em quantidades adequadas e ser de fácil acesso, atrativa e saborosa. De modo que os profissionais nutricionistas compreendem que para o planejamento adequado das dietas, vale atender ao hábito alimentar, contemplar as preferencias, as aversões, e serem consideradas a cultura, a nacionalidade, as questões religiosas e socioeconômicas (Martins; Walder; Rubiatti, 2010).

Outro ponto relevante a considerar é que a Secretaria de Vigilância Sanitária do Ministério da Saúde (ANVISA) no ano de 1999 regulamentou o termo Alimento Funcional “[...] como um alimento ou ingrediente que além das funções nutricionais básicas, quando consumido como parte da dieta usual, produz efeitos metabólicos e/ou efeitos benéficos à saúde, devendo ser seguro para consumo sem supervisão médica” (ANVISA, 1999). Essa regulamentação é de fundamental importância, pois a demanda por produtos funcionais, que exercem efeito específico sobre a dieta devido a sua constituição química, tem sido prioridade de alguns consumidores (Foligné; Daniel; Pot, 2013; Bezerra et al., 2015).

Uma dieta rica em alimentos funcionais promove o bem-estar, disposição e energia, contribuindo para melhoria da qualidade de vida dos indivíduos (Klein; Fassina, 2015). De modo que a introdução destes alimentos funcionais na alimentação pode ser favorável para desenvolver uma microbiota intestinal saudável. Os microrganismos presentes na microbiota estão diretamente envolvidos no sistema imune e regulam as respostas as bactérias patógenas, prevenindo o desenvolvimento de novas doenças, contribuindo para a manutenção e promoção da saúde. A composição da microbiota é obtida no nascimento (especialmente no parto normal, pois, tem contato direto com a microbiota fecal da mãe), e a sua composição final é adquirida por volta dos dois anos de idade, acompanhando o ser humano até o final da sua vida, mas pode ser modificada por diversos fatores, o consumo desses alimentos funcionais modifica e proporciona benefícios a saúde do indivíduo (Barbosa Et Al., 2010; Paixão; Santos, 2016). Além disso, alterações nesse ecossistema bacteriano intestinal podem ocorrer por fatores internos 
ou externos ao hospedeiro, incluindo o uso de antibióticos, de prebióticos e probióticos na alimentação, fatores genéticos, idade, estresse, entre outros (Zhang et al, 2015).

O desequilíbrio ocasionado por esses fatores reflete na modificação da microbiota, com diminuição de bactérias benéficas e aumento de patógenos, caracterizando um quadro de disbiose (Zhang et al, 2015). Esse desequilíbrio da microbiota pode levar a perda de efeitos imunes normais reguladores na mucosa do intestino, sendo associada a um número de doenças inflamatórias e imunomediada. Obter uma homeostase adequada durante o momento de colonização do trato gastrointestinal (TGI) é um dos principais elementos para a modulação do sistema imune adequada e indução da tolerância imunológica. O não funcionamento desse sistema é a grande causa de doenças autoimunes ou atópicas (Satokari et al., 2014; Francino, 2014).

Surge, então, a necessidade de uma nova ótica em nutrição, com a utilização dos alimentos não apenas para saciar a fome e fornecer energia ao corpo, mas também como forma de prevenção ao desenvolvimento de patologias. Nesse contexto, entram em cena os chamados alimentos funcionais, com destaque ao prebióticos, probióticos e simbióticos. A nutrição funcional é uma alternativa para tratamento, prevenção e controle da disbiose intestinal por usar a importância da funcionalidade do trato gastrintestinal como princípio básico, seguido pela especificidade bioquímica, terapêutica voltada ao paciente e não à patologia, estabilidade nutricional, biodisponibilidade de nutrientes e a intervenção de fatores externos à saúde orgânica (Gavanski; Baratto; Gatti, 2015).

A dieta do indivíduo pode ter uma das maiores influências sobre a prevenção e tratamento de doenças crônicas. Uma dieta rica em probióticos ajuda no tratamento de doenças crônicas, os probióticos são microorganismos benefícios responsáveis por conferir vários benefícios ao hospedeiro, como o equilíbrio da microbiota intestinal. Diante do exposto o presente estudo teve como objetivo de avaliar o efeito dos probióticos na prevenção e tratamento de doenças crônicas.

\section{Metodologia}

Trata-se de uma pesquisa de revisão da literatura, de caráter descritivo, qualitativo (Estrela C., 2018). As bases de dados investigadas para a pesquisa foram: Scientific Electronic Library Online (SciELO); Literatura Latino-Americana e do Caribe em Ciências da Saúde - LILACS, Biblioteca Virtual de Saúde (BVS), Google acadêmico, Medline e Pubmed.

Determinaram-se para a execução da pesquisa os descritores específicos e controlados, disponíveis: Probióticos/Probiotics, Probióticos e diabetes/Probiotics and diabetes e Probióticos e câncer/Probiotics and cancer. Durante a etapa de busca, estabeleceram-se os seguintes critérios de inclusão: artigos completos publicados na íntegra, disponíveis nos idiomas inglês, espanhol e português atribuindo-se o recorte temporal nos últimos 20 anos (2000-2020). Inicialmente encontrado 12.000 artigos com o descritor probióticos, ao aplicar o recorte temporal e demais descritores, foi obtido o resultado final de 210 artigos.

Por conseguinte, a análise dos estudos foi feita por meio de uma avaliação criteriosa dos achados na plataforma, com a leitura do título e resumo simples, sondando assim os artigos que realmente se integravam aos critérios de inclusão, metodologia e temática, excluindo-se os fora de contexto, e posteriormente, uma confirmação da análise pela leitura do texto completo (Moher, Liberati, Tetzlaff \& Altman, 2015).

\section{Resultados e Discussão}

\subsection{Probióticos: definições, aplicações clínicas e principais microrganismos utilizados}

A Organização Mundial da Saúde (OMS) define probióticos como microrganismos vivos que, administrados em quantidades adequadas, são capazes de promover benefícios ao hospedeiro a partir da supressão de patógenos endógenos e exógenos, beneficiando a resposta imune (Ganguly et al., 2011). 
Atualmente, os probióticos têm sido amplamente estudados e propagados, com fácil aceitação na alimentação para auxiliar na regulação da flora intestinal (Domingo, 2017). Seus efeitos consistem em estimular a proteção contra bactérias que podem causar danos, como diminuição da permeabilidade intestinal e desempenho inadequado da atividade do sistema imunológico (Paixão; Dos Santos, 2016).

Os probióticos estão no centro dessas descobertas, sendo os principais vetores para promoção de ação nos diferentes microrganismos endógenos humanos, visando ao desenvolvimento do processo de saúde no hospedeiro (Zhu et al., 2015; American, 2018). Esses agentes, que podem estar presentes em alimentos e também em alguns produtos específicos, têm sido utilizados em benefício da saúde e, em alguns casos, no tratamento adjuvante de algumas doenças. As principais cepas utilizadas são dos gêneros Lactobacillus e Bifidobacterium. O histórico de utilização bem como sua regulamentação e seus ganhos sistêmicos encorajaram o uso dessa terapia para diversas doenças associadas ou causadas por bactérias (Cambiaghi et al., 2013).

Os probióticos principais são Lactobacillus acidophilus, Lactobacillus casei, Lactobacillus plantarum, Lactobacillus reuteri, Lactobacillus rhamnosus, Lactobacillus paraca sei, Bifidobacterium bifidum, Bifidobacterium breve, Bifidobacterium infantis, Bifidobacterium lactis, Bifidobacterium longum, Bifidobacterium adolescentis, Saccharomyces bourlardii, Propionibacterium freudenreichii. São considerados também Escherichia, Enterococcus e Bacillus e o fungo Saccaromyces boulardii. No entanto, apesar de serem benéficos ao organismo e de serem frequentemente adicionados à alimentação infantil, os Lactobacillus bulgaricus e Streptococcus thermophilus não são considerados probióticos (Morais; Jacob, 2006).

Atualmente, os probióticos mais utilizados pelos humanos pertencem principalmente aos seguintes gêneros: Lactobacillus, Bifidobacterium e Lactococus, Estreptococos, Enterococos (Simon, 2005). Os probióticos estão sujeitos aos regulamentos contidos na lei geral dos alimentos, segundo a qual eles devem ser seguros para a saúde humana e animal. Nos EUA, microrganismos usados para consumo devem ter o status GRAS (Geralmente Considerado Seguro), regulamentado pelo FDA (Comida e Administração de Drogas). Na Europa, a EFSA (Autoridade Europeia para a Segurança dos Alimentos) introduziu o termo QPS (Presunção Qualificada de Segurança). O conceito QPS envolve alguns critérios adicionais da avaliação de segurança de suplementos bacterianos, incluindo o histórico de uso seguro e a ausência do risco de resistência adquirida a antibióticos (Anadón; Martínez- Larranaga; Martínez, 2006; Gaggia; Mattarelli; Biavati, 2010).

A Tabela 1 apresenta os microrganismos probióticos contidos em produtos farmacêuticos e como aditivos alimentares. 
Tabela 1. Microrganismos probióticos usados na nutrição humana.

\begin{tabular}{|c|c|c|c|}
\hline Tipo Lactobacillus & $\begin{array}{l}\text { Tipo } \\
\text { Bifidobacterium }\end{array}$ & $\begin{array}{l}\text { Outras bactérias do } \\
\text { ácido láctico }\end{array}$ & Outros microrganismos \\
\hline L. acidophilus (a), * & B. adolescentis (a) & Enterococcus faecium (a) & Bacillus clausii (a), * \\
\hline L. amylovorus (b), * & B. $\operatorname{animalis}(\mathrm{a}) *$ & Lactococcus lactis (b), * & E. coli Nissle 1917 (a), (d) \\
\hline L. $\operatorname{casei}(\mathrm{a}),(\mathrm{b}), *$ & B. bifidum (a) & S. thermophilus (a), (c)* & $\begin{array}{l}\text { S. cerevisiae (boulardi) (a), } \\
\text { (e)* }\end{array}$ \\
\hline L. gasseri (a), * & B. breve (b) & & \\
\hline L. helveticus (a), * & B. infantis (a) & & \\
\hline L. johnsonii (b), * & B. longum (a)* & & \\
\hline L. pentosus (b), * & & & \\
\hline L. plantarum (b), * & & & \\
\hline L. reuteri $(\mathrm{a}), *$ & & & \\
\hline L. rhamnosus (a), (b) * & & & \\
\hline
\end{tabular}

Fonte: AESA, 2013; AESA 2017. LEGENDA: (a) Principalmente como produtos farmacêuticos; (b) principalmente como aditivos alimentares; (c) Streptococcus; (d) Escherichia; (e) Saccharomyces; * Microrganismos QPS (Presunção Qualificada de Segurança).

\subsection{Probióticos e câncer: evidências científicas e mecanismos de ação}

Os probióticos são substâncias produzidos por microrganismos que promovem o crescimento de outros microrganismos. Eles atuam como um suplemento nutricional microbiano e afetam beneficamente a saúde humana. Essas bactérias são capazes de resistir às condições ácidas e biliares e ter efeitos benéficos à saúde, como aderência à mucosa intestinal e produção de substâncias antimicrobianas (Sudhakar Reddy et al., 2011). Bactérias probióticas enfrentam câncer reduzindo os efeitos mutagênicos e genotóxicos. Recentemente, estudos mostraram que a bactéria lactobacillus reduza ocorrência e o número de tumores no cólon, fígado, intestino delgado e tecido mamário (Naderi et al., 2014).

A maioria das evidências de sistemas in vitro e de modelos animais e humanos (in vivo) sugerem que os probióticos podem estimular tanto a resposta imune não específica quanto a específica (Saad, 2006). Acredita-se que esses efeitos sejam mediados por ativação dos macrófagos, por aumento nos níveis de citocinas, por aumento da atividade das células destruidoras naturais (NK - “natural killer") e aumento dos níveis de imunoglobulinas (Soares; Navarro, 2010). Merece destaque o fato de que esses efeitos positivos dos probióticos sobre o sistema imunológico ocorrem sem o desencadeamento de uma resposta inflamatória prejudicial (Oliveira, 2009). Assim, pesquisadores relatam que a regulação da resposta imune da mucosa pelos probióticos, talvez, seja a chave para a manipulação da composição da microbiota e intervenção bem-sucedida em ampla gama de doenças crônicas (Tlaskalová-Hogenová et al., 2004), incluindo câncer e diabetes mellitus.

Existem três mecanismos principais pelos quais as infecções podem causar câncer, facilitando principalmente o início e a promoção da carcinogênese. Em primeiro lugar, o agente infeccioso se torna persistente no hospedeiro, induzindo assim a inflamação crônica. Em segundo lugar, os agentes infecciosos podem transformar diretamente as células inserindo oncogenes ativos no genoma do hospedeiro ou inibindo os genes supressores de tumores. Em terceiro lugar, os agentes infecciosos podem induzir imunossupressão e consequentemente reduzir a vigilância imunológica (Kuper; Adami; Trichopoulos, 2000). Deste modo, para existir a prevenção da carcinogênese através do uso da microbiota torna necessário atingir pelo menos um desses três mecanismos. 
Alguns pesquisadores acreditam que os microrganismos probióticos seriam capazes de proteger o hospedeiro contra atividades carcinogênicas por meio de três mecanismos principais: a inibição das bactérias responsáveis por converter substâncias pré-carcinogênicas em carcinogênicas; inibição direta de formação de células tumorais; e a capacidade de algumas bactérias da microbiota intestinal de ligação e/ou inativação carcinogênica (Damião et al., 2009).

O consumo de probióticos tem efeito protetor contra o desenvolvimento do câncer, que pode ser atribuído a várias explicações biológicas gerais: melhorar o sistema imunológico do hospedeiro, modular o estresse oxidativo e a inflamação ou manter as populações bacterianas saudáveis, de forma que elas superem/suprimam as bactérias que produzem agentes cancerígenos, no entanto, mais especificamente (Kumar et al., 2010; Catana et al., 2011; Gata et al., 2017).

\subsection{Probióticos e diabetes: evidências científicas e mecanismos de ação}

A administração de alguns prebióticos e probióticos podem melhorar as alterações metabólicas associadas à obesidade e diabetes, como resistência à insulina, hiperglicemia, inflamação, dislipidemia ou DHGNA em animais (Bashiardes Et al., 2016). Por exemplo, a administração de probióticos, pode contribuir para uma melhora modesta no controle da glicose no sangue (Festi et al., 2014). Além disso, outros pesquisadores demonstraram que o uso de prebióticos, probióticos e simbióticos está associado a pequenas melhorias no controle lipídico (Sáez-Lara et al., 2016).

Como microrganismos vivos não patogênicos, os probióticos são benéficos para os anfitriões, eles também melhoram o sistema imunológico em muitos órgãos através da formação de linfócitos (Prithy; Yoshinori, 2012), também exibem efeitos antimicrobianos e podem melhorar alguns efeitos da dislipidemia, diabetes tipo 2 e colesterol sérico esterol (Singh et al., 2012). Entre esses efeitos, a propriedade antidiabética dosa a biotecnologia e se tornou um importante foco de pesquisa. Por exemplo a progressão do diabetes mellitus pode ser efetivamente evitada ou atrasado usando vários probióticos (Hung; Tseng; Pan, 2016). Probióticos mostraram capacidades na modulação da composição da microbiota intestinal preservar a integridade da barreira intestinal e regular a inflamação (Aw; Fukuda, 2018).

Estudos recentes provaram que bactérias ácidas e probióticos têm destaque em efeitos positivos em humanos ou animais com diabetes tipo1 e 2. Nos modelos animais com diabetes tipo 2, foi investigado e confirmou a capacidade de Lactobacilos para reduzir o risco de desenvolvimento (Andersson et al., 2010). Como suplementos probióticos, Lactobacillus plantarum DSM 15313 e L gasseri BNR17 são estudados em camundongos modelos induzidos a obesidade e diabetes da dieta lipídica alta, apresentaram efeito anti-diabetes, diminuindo o nível de glicose no sangue e melhora a tolerância sem influência de lipídios e níveis de insulina (Andersson et al., 2010; Yun; Park; Kang, 2009).

Um crescente corpo de evidências sugere que associações favoráveis são existentes entre o consumo de probióticos e o perfil metabólico entre diabéticos (Kasińska; Drzewoski, 2015). No entanto, os mecanismos potenciais subjacentes aos efeitos dos probióticos nos parâmetros relacionados à glicemia não são totalmente compreendidos. Um dos principais mecanismos postulados pode envolver aumento do peptídeo 1 semelhante ao glucagon (GLP-1) secreção de células $L$ enteroendócrinas para melhorar os carboidratos no metabolismo, diminuem a glicotoxicidade e aumentam a sensibilidade à insulina nas células alvo (Tremaroli; Bäckhed, 2012). Outros mecanismos propostos para explicar a ação dos probióticos no diabetes referem-se a anti-inflamatórios, antioxidantes e efeitos imunomoduladores e alteração da expressão de alguns genes envolvidos no diabetes (Kim; Sears, 2010; Balakumar et al., 2016).

Em um estudo in vitro e in vivo sobre a cepa probiótica Lactobacillus acidophilus SJLH001 (La-SJLH001) constatouse que a cepa possui propriedades probióticas, atenuando hiperglicemia e hipercolesterolemia induzidas por dieta rica em gorduras. Nos resultados mostraram a melhoria da homeostase da glicose e colesterol em camundongos obesos induzidos, sendo essas descobertas são importantes para a uma aplicação bem-sucedida de probióticos em prevenções no futuro (Sun et al., 2019). 


\subsection{Avanços e perspectivas futuras}

Recentemente foi constatado que cepas específicas pertencentes a Lactobacillus (L. casei cepa Shirota (LAB13), L. gasseri, L. rhamnosus e L. plantarum, entre outras) e Bifidobacterium (principalmente B. infantis, B. longum, e B. breve B3) têm sido amplamente utilizadas como tratamento probiótico em modelos animais estabelecidos com obesidade, devido à sua falta de patogenicidade e baixo nível de resistência a antibióticos. De fato, mais de $85 \%$ dos estudos relataram que camundongos e ratos alimentados com as cepas de Lactobacillus $s p$ e Bifidobacterium $s p$ acima mencionadas, mostraram menos ganho de peso, acúmulo de gordura e tecido adiposo branco em comparação com animais tratados com placebo.

Uma análise detalhada desses estudos também sugere que os probióticos testados exercem seus efeitos anti-obesidade por meio de mecanismos de ação específicos de espécie e cepa, incluindo mudanças benéficas na microbiota intestinal, menor resistência à insulina ou maior saciedade (Ejtahed et al., 2019).

No tratamento de câncer de colorretal, além do tratamento convencional, a suplementação com probióticos pode ser considerada uma ferramenta para a manutenção e restauração da microbiota intestinal em humanos. Estudos in vitro, em modelos animais, demonstraram benefícios na restauração da homeostase (Miquel et al., 2013; Masood et al., 2011), reduzindo a população de bactérias associadas a doenças do cólon (Hemarajata; Versalovic, 2013), inibindo enzimas pró-carcinogênicas ou estimulando o sistema imunológico do hospedeiro (Uccello et al., 2012). Sua ação também promove uma redução nos efeitos colaterais causados pelo tratamento antineoplásico, como diarreia, mucosite (Levit et al., 2018) e dor neuropática (Castelli et al., 2018; Chang et al., 2018).

Pesquisadores evidenciaram que a suplementação de probióticos reduz a gravidade ou encurta a duração da infecção e mostraram a eficácia de Lactobacillus para o tratamento em humanos com infecção do trato respiratório de origem viral (Berggren et al., 2011; Boge, T. et al., 2009; De Vrese et al., 2006). Entre os suplementos alimentares, novos tratamentos potenciais contra a infecção por COVID-19 podem ser baseados em probióticos (Gao; Chen; Fang, 2020), o que pode não apenas reduzir a colonização por espécies patogênicas, mas também aumentar o crescimento bacteriano comensal no trato respiratório (Lehtoranta; Pitkäranta; Korpela, 2014). As espécies bacterianas comensais podem suprimir o crescimento de patógenos pertencentes à mesma família ou gênero, sugerindo o importante papel das interações bacterianas na manutenção da homeostase (Iwase et al., 2010; Ditz et al., 2020).

\section{Conclusão}

Nos últimos anos, vários relatórios sugeriram que a terapia probiótica oral pode de fato prevenir a pneumonia associada a ventilação (PAV), realizaram uma metanálise que incluiu cinco ensaios clínicos randomizados e concluíram que o uso de probióticos estava associado a uma menor incidência de PAV.

\section{Referências}

American Gut. (2018). http://americangut. org/].

Anadón, A., \& Martínez-larranaga, M. (2006). MARTíNEZ M. Probióticos para nutrição animal na Europa União. Regulamentação e avaliação de segurança. Regul. Toxicol. Pharmacol. 45, 91-95.

Andersson, U., et al. (2010). Probióticos diminuem a glicose plasmática no camundongo C57BL / 6J alimentado com alto teor de gordura. Benef. Microbes. 1: $189-196$.

Anvisa - Agência Nacional de Vigilância Sanitária. Conselho Nacional de Segurança Alimentar e Nutricional - Consea. (1999). https://issuu.com/informecaisan/docs/guiaalimentarpopulacaobrasileira_20.

Autoridade Europeia para a Segurança dos Alimentos (AESA). (2013). O relatório de síntese da União Europeia sobre tendências e fontes dezoonoses, agentes zoonóticos e surtos de origem alimentar em 2011. EFSA J. 3129, 1-250.

Autoridade Europeia para a Segurança dos Alimentos (AESA). (2017). Parecer científico sobre a atualização da lista de QPS recomendados agentes biológicos adicionados intencionalmente a alimentos ou rações, conforme notificado à EFSA (atualização de 2017). EFSA J. 15,1-177. 
Aw, W., \& Fukuda, S. (2018). Entendendo o papel do ecossistema do intestino em diabetes mellitus.J. Diabetes Invest. 9 ,5-12.

Balakumar, M., et al. (2016). Melhoria na tolerância à glicose e sensibilidade à insulina por cepas probióticas de origem intestinal indiana em camundongos C57BL / 6J alimentados com dieta rica em gordura. Eur J Nutr.

Barbosa, K., et al. (2010). Estresse oxidativo: conceito, implicações e fatores moduladores. Revista de Nutrição, 23(4), 629-643.

Bashiardes, S., et al. (2016). Fígado gordo não alcoólico e microbiota intestinal. Mol Metab. 5 (9): $782-794$.

Berggren, A., et al. (2011). Estudo randomizado, duplo-cego e controlado por placebo usando novos lactobacilos probióticos para fortalecer a defesa imunológica do corpo contra infecções virais. Eur J Nutr. 50, pp. $203-210$.

Bezerra, M., et al. (2015). Iogurte congelado caprino produzido com polpa de fruta de jambolã fresca e seca por atomização (Eugenia jambolana Lam) e Bifidobacterium animal subespécie lactis BI-07. Ciência Alimentar e Tecnologia, 1, 1-6.

Boge, T., et al. (2009). Uma bebida láctea fermentada com probióticos melhora a resposta dos anticorpos à vacinação contra a gripe em idosos em dois ensaios clínicos randomizados. Vacina. 27, pp. 5677 - 5684.

Cambiaghi, L., et al. (2013). Os probióticos na odontologia: história, conceitos e aplicações na periodontia. Perionews. 7 (1):18-24.

Castelli, V., et al. (2018). Probiótico DSF neutraliza a dor neuropática induzida por quimioterapia. Oncotarget. 9 (46): $27998-28008$.

Catana, C., et al. (2011). A interleucina-17 é um biomarcador pró-aterogênico? Roma. Arco. Microbiol. Immunol. 70, $124-128$.

Chang, C., et al. (2018). Lactobacillus casei variedade rhamnosus probiótico atenua preventivamente a lesão intestinal induzida por 5-fluorouracil / oxaliplatina em um modelo de câncer colorretal singênico. Fronteiras em Microbiologia. 9: 983.

Damião, A., et al. (2009). Probióticos. In: Waitzberg LD. Nutrição Oral, Enteral e Parenteral na Prática Clínica. (4ª ed.): Editora Atheneu.

De Vrese, M., et al. (2006). As bactérias probióticas reduziram a duração e a gravidade, mas não a incidência de episódios de resfriado comum em um ensaio duplo-cego, randomizado e controlado. Vacina. 24, pp. $6670-6674$.

Ditz, B., et al. (2020). Perfil do microbioma do escarro na DPOC: além da detecção singular de patógenos. Tórax. $75: 338$ - 344.

Domingo, J. (2017). Revisão do papel dos probióticos nas doenças gastrointestinais em adultos. Gastroenterologia e Hepatologia. $40: 417-429$.

Ejtahed, H.-S., et al. (2019). Suplementação de probióticos para o gerenciamento da obesidade: Uma revisão sistemática de estudos com animais e ensaios clínicos. J. Funct. Alimentos. 52: 228-242.

Estrela, C. (2018). Metodologia Científica: Ciência, Ensino, Pesquisa. Editora Artes Médicas.

Festi, D., et al. (2014). Microbiota intestinal e síndrome metabólica. Mundo J Gastroenterol. 20 (43): 16079-16094.

Foligné, B., Daniel, C., \& Pot, B. (2013). Probióticos da pesquisa ao mercado: as possibilidades, riscos e desafios. Opinião Atual em Microbiologia, 16, 284292.

Francino, M. (2014). Desenvolvimento precoce da microbiota intestinal e saúde imune. Patógenos. 3(3), 769-790.

Gaggia, F., Mattarelli, P., \& Biavati, B. (2010). Probióticos e prebióticos na alimentação de animais para produção segura de alimentos. Int. J. Food Microbiol. $141, \mathrm{~S} 15-\mathrm{S} 28$

Ganguly, N., et al. (2011). ICMR-DBT Diretrizes para avaliação de probióticos em alimentos. Indian J Med Res. 134: 22-5.

Gao, Q., Chen, Y., \& Fang, J. (2020). 2019 nova infecção por corona vírus e trato gastrointestinal. J Dig Dis. 21 : 125 - 126.

Gata, V., et al. (2017). Tumor infiltrando linfócitos como fator prognóstico no melanoma maligno. Revisão da literatura. J. BUON. 22 , 592-598.

Gavanski, D., Baratto, I., \& Gatti, R. (2015). Avaliação do hábito intestinal e ingestão de fibras alimentares em uma população de idosos. Revista brasileira de obesidade, nutrição e emagrecimento. São Paulo. 9(49). 3-11.

Hemarajata, P., \& Versalovic, J. (2013). Efeitos dos probióticos na microbiota intestinal: mecanismos de imunomodulação intestinal e neuromodulação. Avanços terapêuticos em Gastroenterologia. 6 (1): 39-51.

Hung, S., Tseng, W., \& Pan, T. (2016). Lactobacillus paracasei subsp. paracasei NTU 101 melhora a tolerância à glicose diminuída induzida por uma dieta rica em gordura e frutose em ratos Sprague-Dawley. J. Funct. Foods. 24, 472-481.

Iwase, T., et al. (2010). Staphylococcus epidermidis Esp inibe a formação de biofilme de Staphylococcus aureus e colonização nasal. Nature. 465 : 346 - 349.

Kasińska, M., \& Drzewoski, J. (2015). Efetividade dos probióticos na diabetes tipo 2: uma meta-análise. Pol Arch Med Wewn. 125 (11): 803 -13.

Kim, J., \& Sears, D. (2010). TLR4 e resistência à insulina. Gastroenterol Res Pract. pii: 212563.

Klein, S., \& Fassina, P. (2015). Relação entre o consumo de alimentos funcionais e alterações fisiológicas em praticantes de atividade física. Caderno Pedagógico. Lajeado, 12(1), 22-35. 
Kollef, M. (2005). O que é pneumonia associada à ventilação mecânica e por que ela é importante? Respir Care. 50 : 714 - 721 ; discussão 721 -724.

Kumar, M., et al. (2010). Atributos de probióticos que previnem o câncer: uma atualização. Int. J. Food Sci. Nutr. 61, 473-496.

Kuper, H., Adami, H., \& Trichopoulos, D. (2000). Infecções como uma das principais causas evitáveis de câncer humano. J. Intern. Med. 248 , $171-183$.

Lehtoranta, L., Pitkäranta, A., \& Korpela, R. (2014). Probióticos em infecções por vírus respiratórios. Eur J Clin Microbiol Infect Dis. 33 : 1289 - 1302.

Levit, R., et al. (2018). Efeito protetor da cepa Lactobacillus plantarum CRL2130 com superprodução de riboflavina na mucosite intestinal em camundongos. Nutrição. 54: 165-72.

Martins, D., Walder, B., \& Rubiatti, A. (2010). Educação nutricional: atuando na formação de hábitos alimentares saudáveis de crianças em idade escolar. Ver Simbiologias. 3(4):86-102.

Masood, M., et al. (2011). Efeitos benéficos das bactérias do ácido lático sobre seres humanos. Microbiologia de revisão crítica. 37: 91-8.

Miquel, S., et al. (2013). Faecalibacterium Prausnitzii e saúde intestinal humana. Opinião atual Microbiologia. 6 (3): $255-61$.

Ministério da Saúde. Secretaria de Atenção à Saúde. Departamento de Atenção Básica. Política Nacional de Alimentação e Nutrição. (1ª ed.) Brasília-DF.

Morais, M., \& Jacob, C. (2006). O papel dos probióticos e prebióticos na prática pediátrica. J Pediatr.82 (5): S189-S197.

Naderi, A., et al. (2014). Estudo dos efeitos antagônicos de cepas de Lactobacillus como probióticos em bactérias multirresistentes (MDR) isoladas de infecções do trato urinário (UTIs). Jornal Iraniano de Ciências Médicas Básicas. 17 (3): 201.

Oliveira, A. (2009). O papel dos simbióticos na prevenção, tratamento e modulação da resposta inflamatória em pacientes com carcinoma colorretal. Revista interdisciplinar de estudos experimentais. 1 (4):23-31.

Paixão, L., \& dos Santos, F. (2016). Colonização da microbiota intestinal e sua influência na saúde do hospedeiro. Universitas: Ciências da Saúde, 14(1), 8596.

Prithy, R., \& Yoshinori, M. (2012). Avanços recentes no papel de probióticos na inflamação humana e na saúde intestinal, J. Agric.Food Chem. 60, 82498256.

Sáez-lara, M., et al. (2016). Efeitos de probióticos e simbióticos na obesidade, síndrome de resistência à insulina, diabetes tipo 2 e doença hepática gordurosa não alcoólica: uma revisão de ensaios clínicos em humanos. Int J Mol. Sci. 17 (6).

Satokari, R., et al. (2014). Transplante fecal Tratamento da colite não-infecciosa induzida por antibióticos e acompanhamento da microbiota a longo prazo. Relatos de Casos em Medicina, Nova York, 2014(913867), 1-7, nov.

Siempos, I., Ntaidou, T., \& Falagas, M. (2010). Impacto da administração de probióticos na incidência de pneumonia associada à ventilação mecânica: uma meta-análise de ensaios clínicos randomizados. Crit Care Med. 38: 954 - 962.

Simon, O. (2005). Microrganismos como aditivos para a alimentação animal - Probióticos. Adv. Porco Prod. 16, $161-167$.

Singh, V., et al. (2012). Lactobacillus acidophilus regula positivamente a expressão e função intestinal de NHE3, alt. J. Physiol: Gastrointest. Physiol of the liver. 303, G1393-G1401.

Sudhakar, R., et al. (2011). Bactérias na saúde bucal - probióticos e prebióticos uma revisão. Jornal Internacional de Pesquisa Biológica e Médica. 2 (4): 1226-1233.

Sun, Q., et al. (2019). Análise de mecanismo de glicose melhorada homeostase e metabolismo do colesterol em ratos obesos induzidos com alto teor de gordura tratados com La-SJLH001 via transcriptômica e culturômica. Food Funct. 10, 3556.

Theodorakopoulou, M., et al. (2013). Controvérsias no manejo do doente crítico: o papel dos probióticos. Int J Antimicrob Agents. 42 : S41 - S44.

Tlaskalová-Hogenová, H., et al. (2004). Bactérias comensais (microflora normal), imunidade da mucosa e doenças inflamatórias e autoimunes crônicas. Immunol Lett. 93 (2-3): 97-108.

Toloni, M., et al. (2011). Introdução de alimentos industrializados e de alimentos de uso tradicional na dieta de crianças de creches públicas no município de São Paulo. Revista de Nutrição de Campinas, São Paulo, 24(1), 61-70, jan./fev.

Tremaroli, V., \& Bäckhed, F. (2012). Interações funcionais entre a microbiota intestinal e o metabolismo do hospedeiro. Natureza. 489 (7415): $242-9$.

Uccello, M., et al. (2012). Papel potencial dos probióticos na prevenção do câncer colorretal. BMC Cirurgia. 12 (Supl.1): S35.

Yun, S., Park, H., \& Kang, J. (2009). Efeito de Lactobacillus gasseri BNR17 nos níveis sanguíneos de glicose e peso corporal em um rato modelo de diabetes tipo 2. J Appl Microbiol. 107: 1681-1686.

Zhang, Y., et al. (2015). Impactos das bactérias intestinais na saúde e doenças humanas. Revista Internacional de Ciências Moleculares. 16(4), 7493-7519.

Zhu, A., et al. (2015). Diferenças interindividuais no conteúdo gênico de espécies bacterianas do intestino humano. Genoma Biol. 16:82.

Saad, S. (2006). Probióticos e prebióticos: o estado da arte. Revista Brasileira de Ciências Farmacêuticas. São Paulo. 42(1). 1-16. 
Research, Society and Development, v. 10, n. 5, e30810514930, 2021

(CC BY 4.0) | ISSN 2525-3409 | DOI: http://dx.doi.org/10.33448/rsd-v10i5.14930

Soares, B., \& Navarro, A. (2010). Avaliação dos níveis séricos de imunoglobulina a e consequente resposta do sistema imunológico mediante a suplementação de probióticos e a prática de natação. Revista Brasileira de Nutrição Esportiva, São Paulo. 4(24). 494-501. 